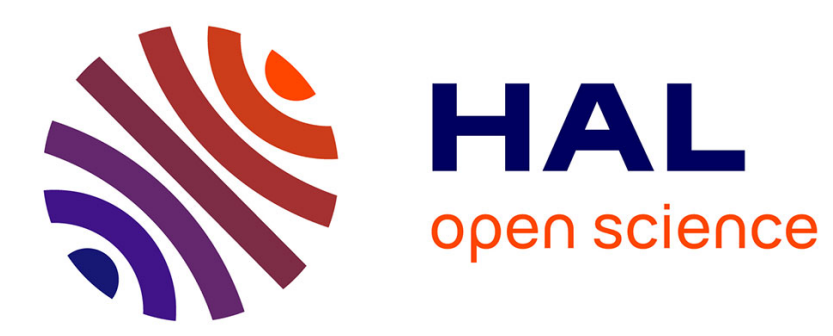

\title{
Digital Two-dimensional Bijective Reflection and Associated Rotation
}

Éric Andrès, Mousumi Dutt, Arindam Biswas, Gaëlle Largeteau-Skapin, Rita Zrour

\section{- To cite this version:}

Éric Andrès, Mousumi Dutt, Arindam Biswas, Gaëlle Largeteau-Skapin, Rita Zrour. Digital Twodimensional Bijective Reflection and Associated Rotation. 2018. hal-01900147

\section{HAL Id: hal-01900147 https://hal.science/hal-01900147}

Preprint submitted on 21 Oct 2018

HAL is a multi-disciplinary open access archive for the deposit and dissemination of scientific research documents, whether they are published or not. The documents may come from teaching and research institutions in France or abroad, or from public or private research centers.
L'archive ouverte pluridisciplinaire HAL, est destinée au dépôt et à la diffusion de documents scientifiques de niveau recherche, publiés ou non, émanant des établissements d'enseignement et de recherche français ou étrangers, des laboratoires publics ou privés. 


\title{
DRAII'
}

\section{Digital Two-dimensional Bijective Reflection and Associated Rotation}

\author{
Eric Andres ${ }^{1 \star}$, Mousumi Dutt ${ }^{2,3}$, Arindam Biswas ${ }^{3}$, Gaelle Largeteau-Skapin ${ }^{1}$, \\ and Rita Zrour ${ }^{1}$ \\ 1 University of Poitiers, Laboratory XLIM, ASALI, UMR CNRS 7252, BP 30179, F-86962 \\ Futuroscope Chasseneuil, France, eric.andres@univ-poitiers.fr \\ 2 Department of Computer Science and Engineering, St. Thomas' College of Engineering and \\ Technology, Kolkata, India, duttmousumi@gmail.com \\ 3 Department of Information Technology, Indian of Engineering Science and Technology, Shibpur, \\ Howrah, India, barindam@gmail.com
}

\begin{abstract}
In this paper, a new bijective reflection algorithm in two dimensions is proposed along with an associated rotation. The reflection line is defined by an arbitrary Euclidean point and a straight line passing through this point. The reflection line is digitized and the $2 \mathrm{D}$ space is paved by digital perpendicular (to the reflection line) straight lines. For each perpendicular line, digital points are reflected by central symmetry with respect to the reflection line. Two consecutive digital reflections are combined to define a digital bijective rotation about arbitrary center (i.e. bijective digital rigid motion).
\end{abstract}

Key words: Digital Reflection; Bijective Digital Rotation; Digital Rotation; Bijective digital rigid motion

\section{Introduction}

Reflection transformation is a one of the most basic linear transforms [1] with many potential applications. In particular, they are key to defining $n$-dimensional rotations [2] and can be used in applications where reflections may play a key role such as lightwave simulations [3-5]. There is however surprisingly few works that deal with the problem such a transform in the digital world. The goal of this paper is to propose an algorithm that performs a digital bijective reflection on a two-dimensional image and to use this to propose a digital bijective rotation algorithm that works for an arbitrary rotation center (i.e. bijective digital rigid motion. See [6] for a first algorithm and a theoretical discussion on the subject of digital rigid motions). While the reflection transform in the digital world has not been studied much, digital rotations and more precisely bijective digital rotations has been a point of interest of the digital community for some years now [7]. The characterization of a discretized rotation is stated in [8] which is the composition of an Euclidean rotation with rounding operations. Using Gaussian integers the bijective discrete rotation is characterized in [9]. In [10], an incremental approach to discretized rotations is explained. In [11], bijective rigid motion in $2 \mathrm{D}$ cartesian

\footnotetext{
* Corresponding Author
} 
grid is stated. Other mentionable work on characterization of bijective rotations in $3 \mathrm{D}$ space is [12]. The bijective rotation is also studied on hexagonal grid in discrete space [13].

A continuous tranform applied to a digital image is not, in general, bijective, nor injective or surjective for that matter, even for an apparently very simple transform such as the reflection transform (see Figure 1). Our idea is to digitize the Euclidean reflection straight line as naive digital lines (i.e. 8-connected digital lines) and to partition the two-dimensional space into naive digital lines that are perpendicular to the reflection line. A central symmetry is performed on the points of each Perpendicular Digital Straight Line (PDSL) according to the Digital Reflection Straight Line (DRSL). This is always possible because we can compute the exact pixel to pixel correspondance on each side of the DRSL for such naive digital lines. The main problem comes from the fact that the digital reflection line and a given digital perpendicular line may or may not intersect and so two different cases have to be considered.

The organization of the paper is as follows: in Sec. 2, we present the preliminaries. Section 3 presents the mathematical foundation of bijective digital reflection. The bijective digital rotation about an arbitrary center can be determined by applying digital reflection twice (see Sec. 4). An error criteria based on the distance between the continuous and the digital rotated points are presented in Sec. 4.2. The concluding remarks are presented in Sec. 5.

\section{Preliminaries}

Let $\{\boldsymbol{i}, \boldsymbol{j}\}$ denote the canonical basis of the 2-dimensional Euclidean vector space. Let $\mathbb{Z}^{2}$ be the subset of $\mathbb{R}^{2}$ that consists of all the integer coordinate points. A digital (resp. Euclidean) point is an element of $\mathbb{Z}^{2}$ (resp. $\mathbb{R}^{2}$ ). Two digital points $p\left(p_{x}, p_{y}\right)$ and $q\left(q_{x}, q_{y}\right)$ are said to be 8-connected if $\left|p_{x}-q_{x}\right| \leqslant 1$ and $\left|p_{y}-q_{y}\right| \leqslant 1$.

For $x \in \mathbb{R},\lfloor x\rfloor$ is the biggest integer smaller or equal to $x$ and $\lceil x\rceil$ is the smallest integer greater or equal to $x$.

A naive digital straight line is defined as all the digital points verifying $-\frac{\max (|a|,|b|)}{2} \leqslant a x-b y<\frac{\max (|a|,|b|)}{2}$, where $\frac{a}{b}$ represents the slope of the Digital Straight Line (DSL) and $\omega=\max (|a|,|b|)$ the arithmetical thickness [14]. A naive DSL is 8-connected such that if you remove any point of the line then it is not 8-connected anymore [14]. There are no simple points.

A reflection transformation $\mathcal{R}_{\theta,\left(x_{o}, y_{o}\right)}: \mathbb{R}^{2} \mapsto \mathbb{R}^{2}$ reflects (or flips) a continuous point, like in a mirror, on a continuous straight line called the reflection line. The reflection line is defined as the line passing through a point of coordinates $\left(x_{o}, y_{o}\right) \in \mathbb{R}^{2}$ and the vector director $v=(\sin \theta, \cos \theta)$. The corresponding digital reflection is denoted $R_{\theta,\left(x_{o}, y_{o}\right)}: \mathbb{Z}^{2} \mapsto \mathbb{Z}^{2}$. A continuous rotation $\mathcal{R}_{\theta} t_{\theta,\left(x_{o}, y_{o}\right)}$ of center $\left(x_{o}, y_{o}\right)$ and angle $\theta$ can be defined as the composition of two continuous reflections $\mathcal{R}_{\alpha,\left(x_{o}, y_{o}\right)}$ and $\mathcal{R}_{\alpha+\frac{\theta}{2},\left(x_{o}, y_{o}\right)}$. 


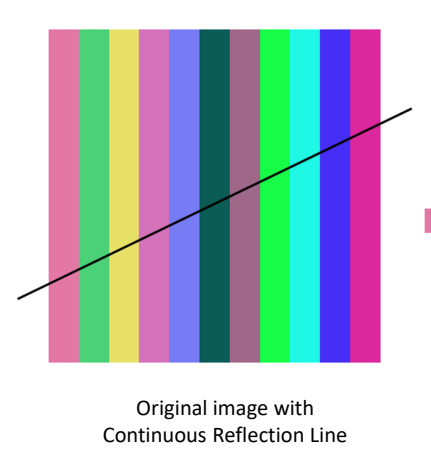

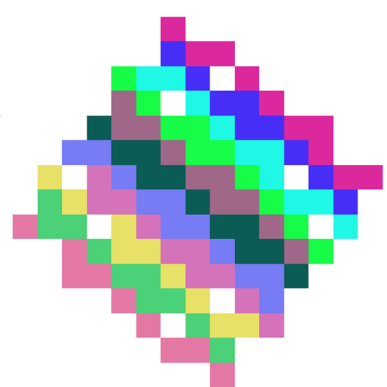

Discretized Image Reflection

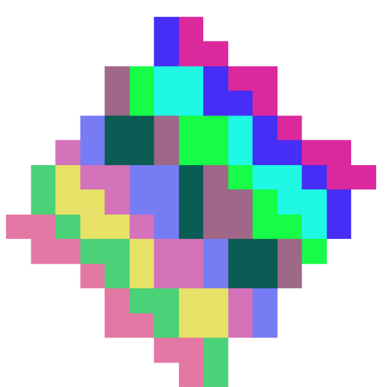

Bijective Digital Reflection

Fig. 1. Reflection Transform applied to an image. On the left, the original image. In the center, the continuous reflection transform composed with a rounding function. On the right, our proposed bijective digital reflection

\section{Digital Reflection}

\subsection{Principle}

Let us consider the continuous reflection transform, $\mathcal{R}_{\theta,\left(x_{o}, y_{o}\right)}$. It is not difficult to note that if we simply compose the continuous reflection with a digitization transform (such as for instance $\mathcal{D}:(x, y) \mapsto(\lfloor x+0.5\rfloor,\lfloor y+0.5\rfloor))$ then we have a transform that is, in general, neither injective, surjective and of course not bijective (see the middle image in Figure 1).

To avoid this problem and create a digital bijective reflection transform, a completely digital framework is proposed here based on the following digital primitives:

- A naive DSL called digital reflection straight line (DRSL);

- and a partition of the digital space with naive DSLs that are perpendicular to the digital reflection straight line. These digital lines are called Perpendicular Digital Straight Lines (PDSL).

The idea of the reflection method is the following: let us consider a point $p$ that belongs to a given digital perpendicular straight line $P_{k}$ ( $k$ is a parameter identifying the PDSL in the partition). The point $p$ may belong to the digital reflection line (in case there is an intersection) or lie on either side of that line. Let the digital reflection of $p$ be determined in the following way (see Figure 2):

- let us first suppose that $p$ lies in the intersection between the PDSL $P_{k}$ and the DRSL. Such an intersection does not always exist between the two digital lines but when it does (in this case point $p$ ) then the image of $p$ by the digital reflection is $p$; 

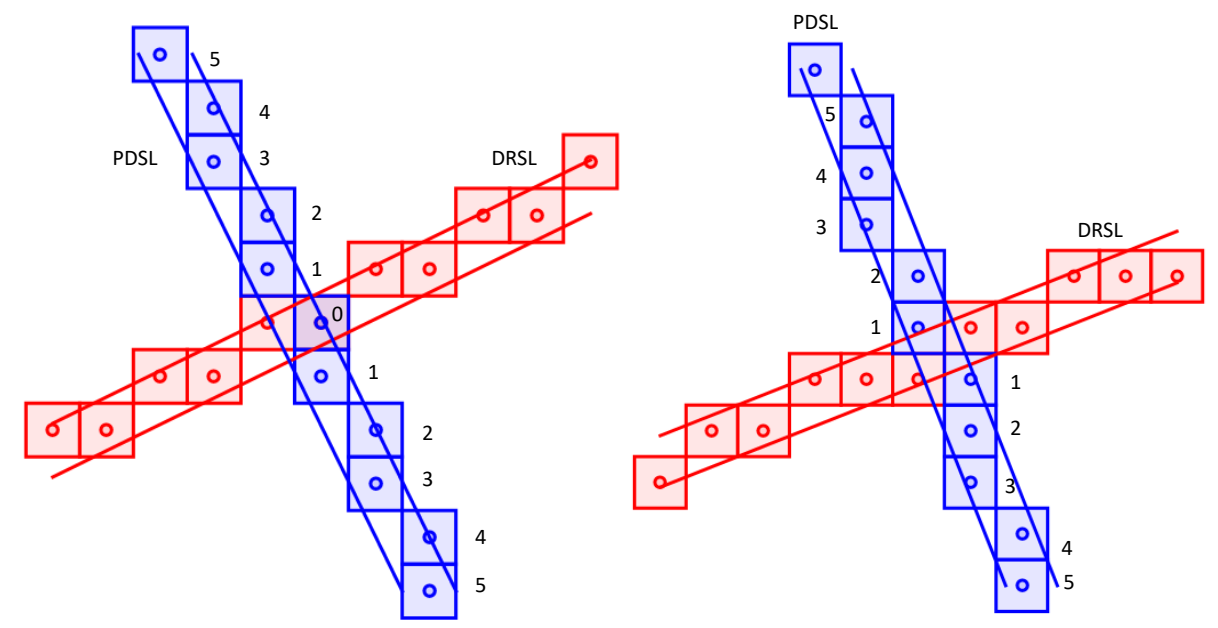

Fig. 2. The image of each point is the mirror image in the perpendicular line according to the digital reflection straight line. The image of a point numbered $i$ is the opposite point numbered $i$. The point numbered 0 is its own image.

- Let us now suppose that $p$ does not belong to the intersection between the PDSL and the DRSL (whether such an intersection exists or not). The point $p$ is then located on one side of the DRSL: the digital space is split by the DRSL into three regions, the DRSL and two regions on each side of it. All the digital points of $P_{k}$ on each side of the DRSL can formally be ordered according to the distance to the DRSL with as first point the closest one to the DRSL. As we will see, there actually is no real ordering needed. Let us suppose that $p$ is the $n^{\text {th }}$ point of the ordered list on its side and a digital point $q$ is the $n^{\text {th }}$ point of the list on the other side of the DRSL. The digital reflection of $p$ will then be the point $q$ and vice-versa.

Figure 2 shows the two possible cases: on the left, the perpendicular digital line has an intersection point (the point marked as point 0) with the digital reflection line, while on the right there is no intersection point. The points marked by the number $i$ (from 1 to 5 ) are images of each other. The image of the point marked 0 is itself. The idea is to create a digital transformation that is bijective and easily reversible. Let us now, in the next section, present the various definitions and mathematical details to make this work out.

\subsection{Mathematical Details}

The mathematical details of the works is stated here. Let us consider the continuous reflection $\mathcal{L}$ line defined by the continuous point $\left(x_{o}, y_{o}\right) \in \mathbb{R}^{2}$ and the 
direction vector $v=(a, b)=(\sin \theta, \cos \theta)$. The analytical equation of the reflection line is given by: $\mathcal{L}=\left\{(x, y) \in \mathbb{R}^{2}: a\left(x-x_{o}\right)-b\left(y-y_{o}\right)=0\right\}$.

The digital reflection straight line $(D R S L)$ the digitization (as naive DSL) of $\mathcal{L}$ defined as all the digital points verifying:

$$
D R S L:-\frac{\max (|a|,|b|)}{2} \leqslant a\left(x-x_{o}\right)-b\left(y-y_{o}\right)<\frac{\max (|a|,|b|)}{2}
$$

The perpendicular digital straight lines (PDSL) $P_{k}$ are naive DSLs defined as all the digital points $(x, y)$ verifying :

$$
P_{k}: \frac{(2 k-1) \max (|a|,|b|)}{2} \leqslant b\left(x-x_{o}\right)+a\left(y-y_{o}\right)<\frac{(2 k+1) \max (|a|,|b|)}{2}
$$

It is easy to see that all the PDSLs are perpendicular to the DRSL and that the set of PDSLs, as a set of naive digital lines, partitions the two dimensional digital space.

Let us now suppose for what follows, w.l.o.g, that we have $-\pi / 4 \leqslant \theta \leqslant \pi / 4$, then $0 \leqslant|a| \leqslant b$ and thus $\max (|a|,|b|)=b$. The DRSL is then defined by $-\frac{b}{2} \leqslant a\left(x-x_{o}\right)-b\left(y-y_{o}\right)<\frac{b}{2}$ and the PDSLs are then defined by $P_{k}$ : $\frac{(2 k-1) b}{2} \leqslant b\left(x-x_{o}\right)+a\left(y-y_{o}\right)<\frac{(2 k+1) b}{2}$.

The method supposes that we are able to formally order the points of a given perpendicular line that are located on either side of the reflection line. This is always possible because the PDSLs are naive lines and for an angle verifying $-\pi / 4 \leqslant \theta \leqslant \pi / 4$, there is one and only one point per integer ordinate $y$ (For angles $\pi / 4 \leqslant \theta \leqslant 3 \pi / 4$, we will have, symmetrically, one and only one point per integer abscissa $x$ in a given PDSL).

More precisely, $\frac{(2 k-1) b}{2} \leqslant b\left(x-x_{o}\right)+a\left(y-y_{o}\right)<\frac{(2 k+1) b}{2}$ means that $\frac{2 k-1}{2}+$ $x_{o}-\frac{a}{b}\left(y-y_{o}\right) \leqslant x<\frac{2 k+1}{2}+x_{o}-\frac{a}{b}\left(y-y_{o}\right)$. Since $\frac{2 k+1}{2}-\frac{2 k-1}{2}=1$, there is one and only one value $x$. For a given ordinate $y$, the abscissa $x$ in the PDSL $P_{k}$ is given by the following function:

$$
\mathcal{X}(y)=\left\lceil\frac{(2 k-1)}{2}+x_{o}-(a / b)\left(y-y_{o}\right)\right\rceil
$$

Let us note that for a given digital point $p(x, y)$, it is easy to determine the PDSL $P_{k}$ it belongs to: $\frac{(2 k-1) b}{2} \leqslant b\left(x-x_{o}\right)+a\left(y-y_{o}\right)<\frac{(2 k+1) b}{2}$ leads to $2 k \leqslant 2 \frac{b\left(x-x_{o}\right)+a\left(y-y_{o}\right)}{b}+1<2 k+2$ and thus:

$$
k=\left\lfloor\left(x-x_{o}\right)+\frac{a}{b}\left(y-y_{o}\right)+\frac{1}{2}\right\rfloor
$$

The next question that arises is the determination and the localization of the potential intersection point between the digital reflection line and a given digital perpendicular line. As already mentioned, there may be 0 or 1 digital intersection points between the DRSL and a given PDSL since both are naive digital lines. We propose here a simple criterion to determine the existence of 


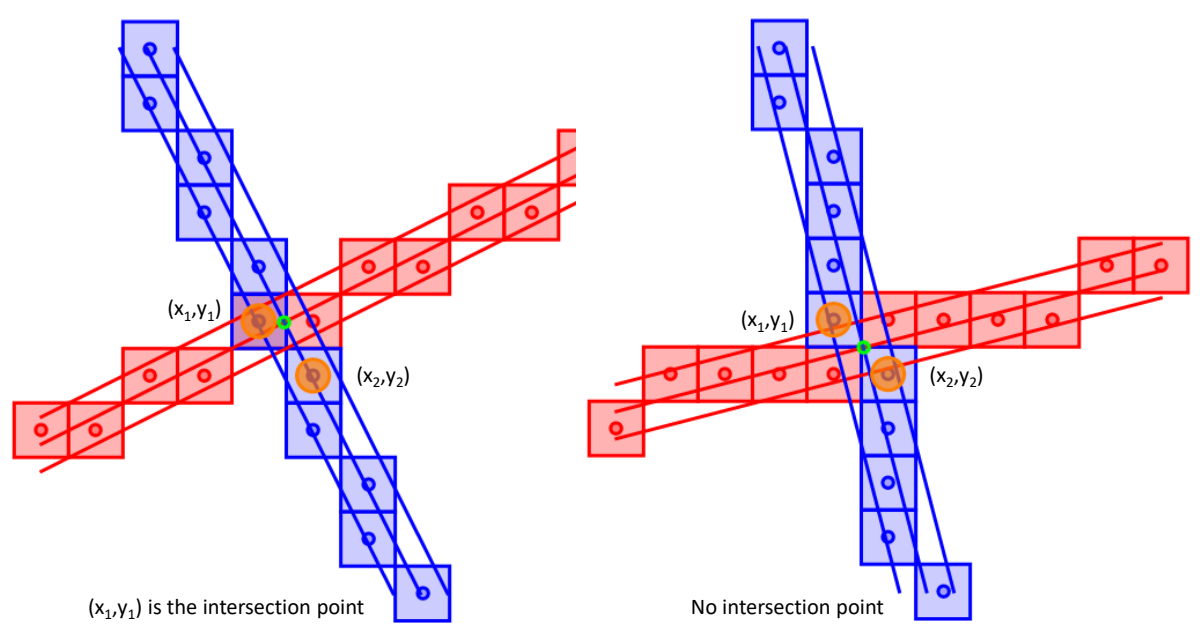

Fig. 3. The green point represents the intersection point between the continuous reflection line and the continuous perpendicular line. The orange disks mark the two candidate points for the digital intersection. On the left $\left(x_{1}, y_{1}\right)$ is the digital intersection point between the DRSL and the PDSL. On the right there is no digital intersection point.

such an intersection point and its localization, if it exists. If the point does not exist, it will yield the closest point of the PDSL to the DRSL on either side of the digital reflection line.

Firstly, let us note that the DRSL is the digitization of the continuous reflection line of equation: $a\left(x-x_{o}\right)-b\left(y-y_{o}\right)=0$ which lies in the middle of the strip defining the DRSL. In the same way, the continuous straight line of equation $b\left(x-x_{o}\right)+a\left(y-y_{o}\right)=k b$ lies in the middle of the strip defining the perpendicular digital straight line $P_{k}$. The intersection point of those two continuous lines is given by $\left(k b^{2}+x_{o}, a b k+y_{o}\right)\left(\right.$ since $\left.a^{2}+b^{2}=1\right)$. It is easy to understand that the only digital intersection point, if it exists, has an ordinate value given by either $\left\lceil a b k+y_{o}\right\rceil$ or $\left\lfloor a b k+y_{o}\right\rfloor$. This is a direct consequence of the fact that the DRSL is a naive digital line of slope between -1 and 1 and thus with only one digital point per integer ordinate value. We have therefore a very simple test to determine the existence and coordinates of the intersection point (see Figure 3):

- let us define $\left(x_{1}, y_{1}\right)=\left(\mathcal{X}\left(\left\lceil a b k+y_{o}\right\rceil\right),\left\lceil a b k+y_{o}\right\rceil\right)$ and $\left(x_{2}, y_{2}\right)=\left(\mathcal{X}\left(\left\lfloor a b k+y_{o}\right\rfloor\right),\left\lfloor a b k+y_{o}\right\rfloor\right)$

- If $\left(x_{1}, y_{1}\right)$ belongs to the DRSL then there is an intersection point, $\left(x_{1}, y_{1}\right)$ in this case. A central symmetry of the PDSL points can be performed around the ordinate value $y_{1}$. The digital reflection of a point $p\left(x_{p}, y_{p}\right) \in P_{k}$ is given by $\left(\mathcal{X}\left(2 y_{1}-y_{p}\right), 2 y_{1}-y_{p}\right)$. Let us note here that the central symmetry 


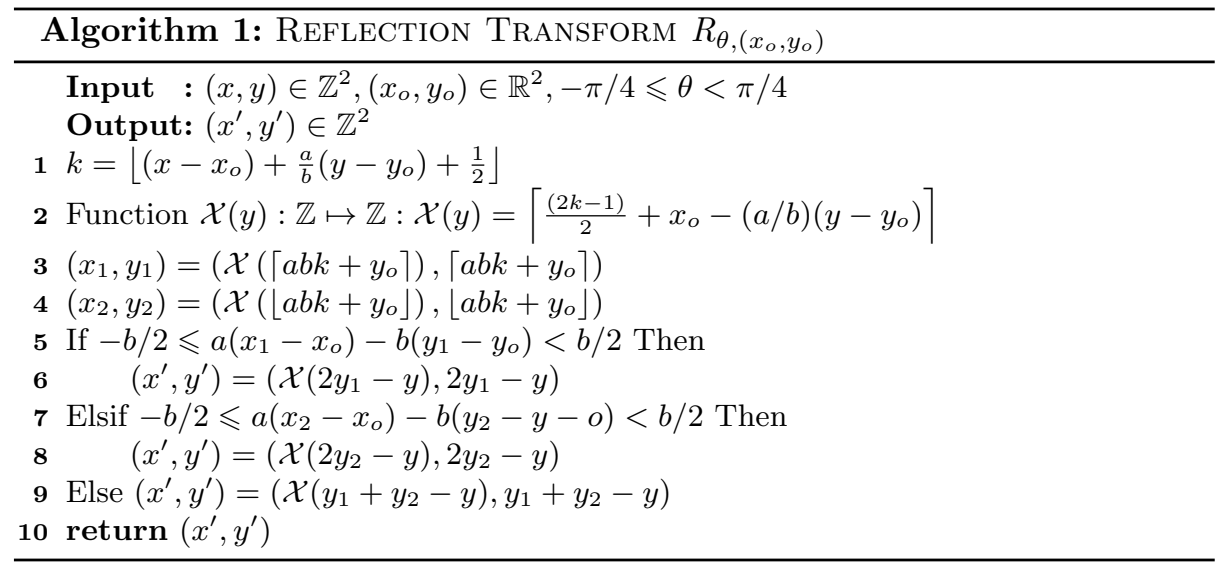

around the ordinate $y_{1}$ does not mean here that there is a central symmetry around the point $\left(x_{1}, y_{1}\right)$ because space partition would not be guaranteed and thus bijectivity would be lost. The abcissa $\mathcal{X}\left(2 y_{1}-y_{p}\right)$ is computed so that the reflected point still belongs to $P_{k}$.

- Else if $\left(x_{2}, y_{2}\right)$ belongs to the the DRSL then there is an intersection point, $\left(x_{2}, y_{2}\right)$. A central symmetry of the PDSL points can be performed around the ordinate value $y_{2}$. The digital reflection of a point $p\left(x_{p}, y_{p}\right) \in P_{k}$ is given by $\left(\mathcal{X}\left(2 y_{2}-y_{p}\right), 2 y_{2}-y_{p}\right)$.

- Otherwise there is no intersection point and $\left(x_{1}, y_{1}\right)$ and $\left(x_{2}, y_{2}\right)$ are the first points of the PDSL on each side of the Reflection line. The central symmetry can be performed around the ordinate value $\frac{y_{1}+y_{2}}{2}$. The digital reflection of a point $p\left(x_{p}, y_{p}\right) \in P_{k}$ is given by $\left(\mathcal{X}\left(y_{1}+y_{2}-y_{p}\right), y_{1}+y_{2}-y_{p}\right)$.

The important point here is that, since there is only one point per ordinate $y$, a central symmetry on the ordinate leads directly to the reflection point. Algorithm 1 presents the digital reflection transform method.

\subsection{Bijectivity of the Digital Reflection Transform}

Algorithm 1 provides a digital reflection method for a digital point. It is not very difficult to see that this defines a bijective digital reflection transform. Let us briefly summarizes the arguments for this:

- The computation of the value $k$ in line 1 of algorithm 1 yields the same value for any point of a given PDSL (and only for those).

- Line 6, 8 and 9 ensure that the image of a digital point of a given PDSL $P_{k}$ is a digital point belonging to $P_{k}$. Indeed the ordinate values $2 y_{1}-y, 2 y_{2}-y$ and $y_{1}+y_{2}-y$ are integers if $y_{1}, y_{2}$ and $y$ are integers and $(\mathcal{X}(y), y)$ is, by construction, a point of $P_{k}$ if $y$ is an integer.

- The reflection of the reflection of a digital point $(x, y) \in P_{k}$ is the digital point $(x, y)$ : Let us consider $(x, y) \mapsto\left(\mathcal{X}\left(y^{\prime}\right), y^{\prime}\right) \mapsto\left(\mathcal{X}\left(y^{\prime \prime}\right), y^{\prime \prime}\right)$ where $y^{\prime}=2 y_{1}-y$, 
$y^{\prime}=2 y_{2}-y$ or $y^{\prime}=y_{1}+y_{2}-y$. Let us note first that $(x, y)=(\mathcal{X}(y), y)$. For all three cases, we have respectively $y^{\prime \prime}=2 y_{1}-y^{\prime}=2 y-1-\left(2 y_{1}-y\right)=y, y^{\prime \prime}=$ $2 y_{2}-y^{\prime}=2 y_{2}-\left(2 y_{2}-y\right)=y$ and $y^{\prime \prime}=y_{1}+y_{2}-y^{\prime}=y_{1}+y_{2}-\left(y_{1}+y_{2}-y\right)=y$ which proves the point.

\section{Reflection Based Rotation}

As mentioned in the preliminaries, a continuous rotation transform $\operatorname{Rot}_{\theta,\left(x_{o}, y_{o}\right)}$ of center $\left(x_{o}, y_{o}\right)$ and angle $\theta$ can be defined as the composition of two reflections based on two reflection lines passing through $\left(x_{o}, y_{o}\right)$ with an angle $\theta / 2$ between the two lines. In the same way, a digital rotation:

$$
\operatorname{Rot}_{\theta,\left(x_{o}, y_{o}\right)}(x, y)=\left(R_{\alpha+\frac{\theta}{2},\left(x_{o}, y_{o}\right)} \circ R_{\alpha,\left(x_{o}, y_{o}\right)}\right)(x, y)
$$

Let us note right away that, since the digital reflection transform is bijective, the digital rotation based on the reflection transforms will be bijective as well. Furthermore, the inverse transform is easily defined. This last point may seem obvious but it is not because a digital transform is bijective that the inverse transform is easily computed.

Compared to previous digital rotations methods $[7,15,13,10]$, there is an extra parameter that comes into play: the angle $\alpha$.

\subsection{Rotation Evaluation Criteria}

In order to evaluate the "quality" of such a digital rotation, let us present some simple error measures [7]. Each grid point has one and only one image through a bijective digital rotation but that does not mean that the digital rotation is a good approximation of the continuous one. To measure how "wrong" we are by choosing the digital rotation over the continuous one, we are considering two distance criteria $([7])$. Let us denote $\mathcal{R}_{\theta} t_{\theta\left(x_{o}, y_{o}\right)}(p)$ the continuous rotation of center $\left(x_{o}, y_{o}\right)$ and angle $\theta$ of a grid point $p \in \mathbb{Z}^{2}$ and $\operatorname{Rot}_{\theta,\left(x_{o}, y_{o}\right)}(p)$ the digital rotation of center $\left(x_{o}, y_{o}\right)$ of a grid point $p$. /par The Maximum Distance quality criteria $(M D)$ consists in computing $\max _{p \in \mathbb{Z}^{2}}\left(d\left(\mathcal{R o t}_{\theta,\left(x_{o}, y_{o}\right)}(p), \operatorname{Rot}_{\theta,\left(x_{o}, y_{o}\right)}(p)\right)\right.$. The average distance quality criteria $(A D)$ consists in computing

$a v g_{p \in \mathbb{Z}^{2}}\left(d\left(\mathcal{R} o t_{\theta\left(x_{o}, y_{o}\right)}(p), \operatorname{Rot}_{\theta\left(x_{o}, y_{o}\right)}(p)\right)\right.$, where $\operatorname{avg}_{p \in \mathbb{Z}^{2}}$ is the average distance over the grid.

The idea is to measure what error is made by using the digital rotation instead of the continuous one in terms of distance to the optimal position (to the continuously rotated point). Let us finish by noting that for the best known digital bijective rotations $[15,9,11]$ and for the angles where the digitized rotations are bijective (except for the trivial $k \pi / 2$ angles), we obtain the best maximum MD values of $\frac{\sqrt{2}}{2} \approx 0.7$ and average distance $\mathrm{AD}$ of $\approx 0.3$. Note also that this is not obtained for all angles. 

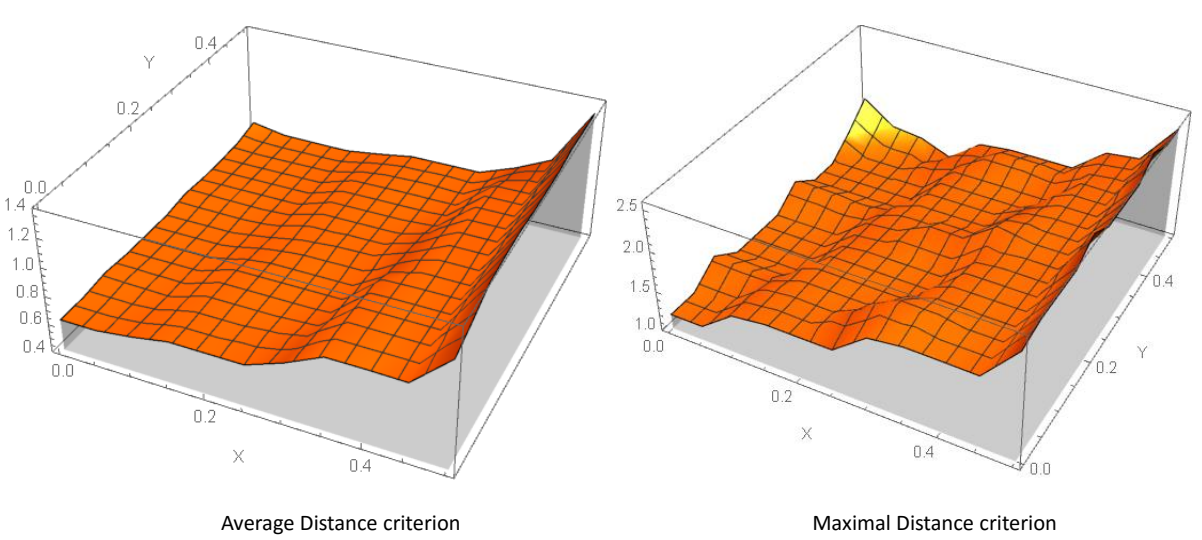

Fig. 4. Rotations of center $(x, y)$, angle $\pi / 8$ and $\alpha=0$

\subsection{Evaluation Analysis}

It is difficult to give a completely detailed rotation evaluation here due to lack of space as there are four different parameters that can influence the outcome of the evaluation criteria: the center coordinates $\left(x_{o}, y_{o}\right)$ and the angles $\theta$ and $\alpha$.

At first, we wanted to have an idea on the effect of the rotation center on the rotation error measure with the angle $\alpha=0$ and $\theta=\pi / 8$. This means that the first reflection corresponds to a reflection with the continuous reflection line $y=y_{o}$, for $-0.5 \leqslant y_{o}<0.5$ and the digital reflection $y=0$. In Figure 4 we represented the influence of moving the center on the interval $[0,0.5]^{2}$. As can be seen, the error measures are almost not affected by the shift on the ordinate but greatly by a shift on the abscissa of the rotation center. This can be explained by the fact that the shift on the perpendicular lines has a direct influence on the lateral error that adds up to the global error. Let us note that the surfaces are similar looking for other couple of angles $(\alpha, \theta)$ even though the amplitude of the error measure may be different.

Next, we wanted to have an idea on the effect of the angle $\alpha$ on the rotation error measures. So, for each rotation angle $0 \leqslant \theta \leqslant \pi / 4$, the average error measures for 500 rotations with randomly chosen center and respectively angle $\alpha=0$ and a randomly chosen angle $0 \leqslant \alpha \leqslant \pi / 4$. The result can be seen in figure 5 . It can be seen that the angle $\alpha$ has only a minor influence on the average error but the maximal error is typically significantly increased for an angle $\alpha>0$. It is interesting to note that this is not always so clear: the digital rotation of angle $\pi / 6$ and center $(0,0)$ has an average / maximum error of $(0.6367,1.3972)$ for $\alpha=0$ and $(0.5726,1.4186)$ for $\alpha=\pi / 6$. What can be noticed as well is that the error ratio between $\alpha=0$ and random chosen $\alpha$ is relatively stable over all the rotation angles. The error measure in general are higher than those obtained for 


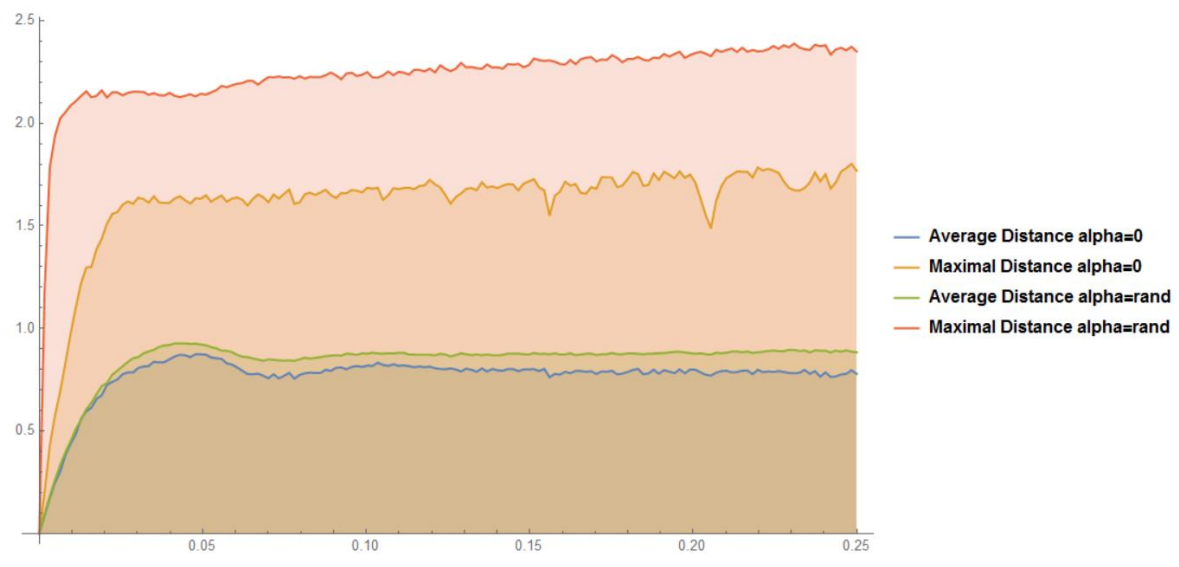

Fig. 5. For each rotation angle $0 \leqslant \theta \leqslant \pi / 4$, the average error measures of 500 Rotations with random center and respectively $\alpha=0$ and random $\alpha$ (with $0 \leqslant \alpha \leqslant \pi / 4$ )

the shear based method [7] that is also defined for all angles and centers. The reflection based rotation is however way easier to implement.

\section{Conclusion}

A novel bijective digital reflection transform in two dimensions has been proposed. The reflection line is defined by an arbitrary Euclidean point and a straight line passing through this point. A rotation can be defined by the composition of two reflections, and so an associated new bijective digital rotation transform has been proposed. This new bijective digital rotation is defined for all angles and for all rotation centers, defining a rigid motion transform (see figure 6 that illustrates the rotation of an image at various angles and centers). In average the distance between a continuously and a digitally rotated point is about 0.8 (for a pixel of side 1). This reflection based rotation seems well suited for extensions to higher dimensions which is not easily done with previous methods. This is the main perspective for the future: exploring digital reflection based bijective rotations in three and higher dimensions.

\section{References}

1. R. Goodman, Alice through looking glass after looking glass: The mathematics of mirrors and kaleidoscopes, The American Mathematical Monthly 111 (4) (2004) 
281-298.

URL http://www.jstor.org/stable/4145238

2. A. Richard, L. Fuchs, G. Largeteau-Skapin, E. Andres, Decomposition of ndrotations: Classification, properties and algorithm, Graphical Models 73 (6) (2011) 346-353.

3. P. S. Heckbert, P. Hanrahan, Beam tracing polygonal objects, ACM SIGGRAPH Computer Graphics 18 (3) (1984) 119-127.

4. R. Zrour, P. Y. Chatelier, F. Feschet, R. Malgouyres, Parallelization of a discrete radiosity method, in: Euro-Par 2006, Parallel Processing, 12th International EuroPar Conference, Dresden, Germany, August 28 - September 1, 2006, Proceedings, 2006, pp. $740-750$.

5. F. Mora, G. Ruillet, E. Andres, R. Vauzelle, Pedagogic discrete visualization of electromagnetic waves, Eurographics poster session, EUROGRAPHICS, Granada, Spain (January 2003).

6. K. Pluta, P. Romon, Y. Kenmochi, N. Passat, Bijective digitized rigid motions on subsets of the plane, Journal of Mathematical Imaging and Vision 59 (1) (2017) 84-105.

7. E. Andres, The quasi-shear rotation, in: Discrete Geometry for Computer Imagery: 6th International Workshop, DGCI'96, Lyon, France, November 13-15, 1996, Proceedings, Vol. 1176, Springer Science \& Business Media, 1996, p. 307.

8. B. Nouvel, E. Rémila, Characterization of bijective discretized rotations, in: Proceedings of the 10th International Conference on Combinatorial Image Analysis, IWCIA'04, Springer-Verlag, Berlin, Heidelberg, 2004, pp. 248-259.

9. T. Roussillon, D. Coeurjolly, Characterization of bijective discretized rotations by gaussian integers, Tech. rep., LIRIS UMR CNRS 5205 (January 2016).

10. B. Nouvel, E. Rémila, Incremental and transitive discrete rotations, in: Proceedings of the 11th International Conference on Combinatorial Image Analysis, IWCIA'06, Springer-Verlag, Berlin, Heidelberg, 2006, pp. 199-213.

11. K. Pluta, P. Romon, Y. Kenmochi, N. Passat, Bijective rigid motions of the $2 \mathrm{~d}$ cartesian grid, in: Proceedings of the 19th IAPR International Conference on Discrete Geometry for Computer Imagery - Volume 9647, DGCI 2016, Springer-Verlag, Berlin, Heidelberg, 2016, pp. 359-371.

12. K. Pluta, P. Romon, Y. Kenmochi, N. Passat, Bijectivity certification of 3d digitized rotations, in: Proceedings of the 6th International workshop on Computer Topology on Image Context, CTIC 2016, 2016, pp. 30-41.

13. K. Pluta, T. Roussillon, D. Coeurjolly, P. Romon, Y. Kenmochi, V. Ostromoukhov, Characterization of bijective digitized rotations on the hexagonal grid, Tech. rep., HAL, submitted to Journal of Mathematical Imaging and Vision. (June 2017). URL https://hal .archives-ouvertes.fr/hal-01540772

14. J.-P. Reveillès, Calcul en nombres entiers et algorithmique, $\mathrm{Ph}$.D. thesis, Université Louis Pasteur, Strasbourg, France (1991).

15. M.-A. Jacob, E. Andres, On discrete rotations, in: Discrete Geometry for Computer Imagery, 1995, p. 161. 

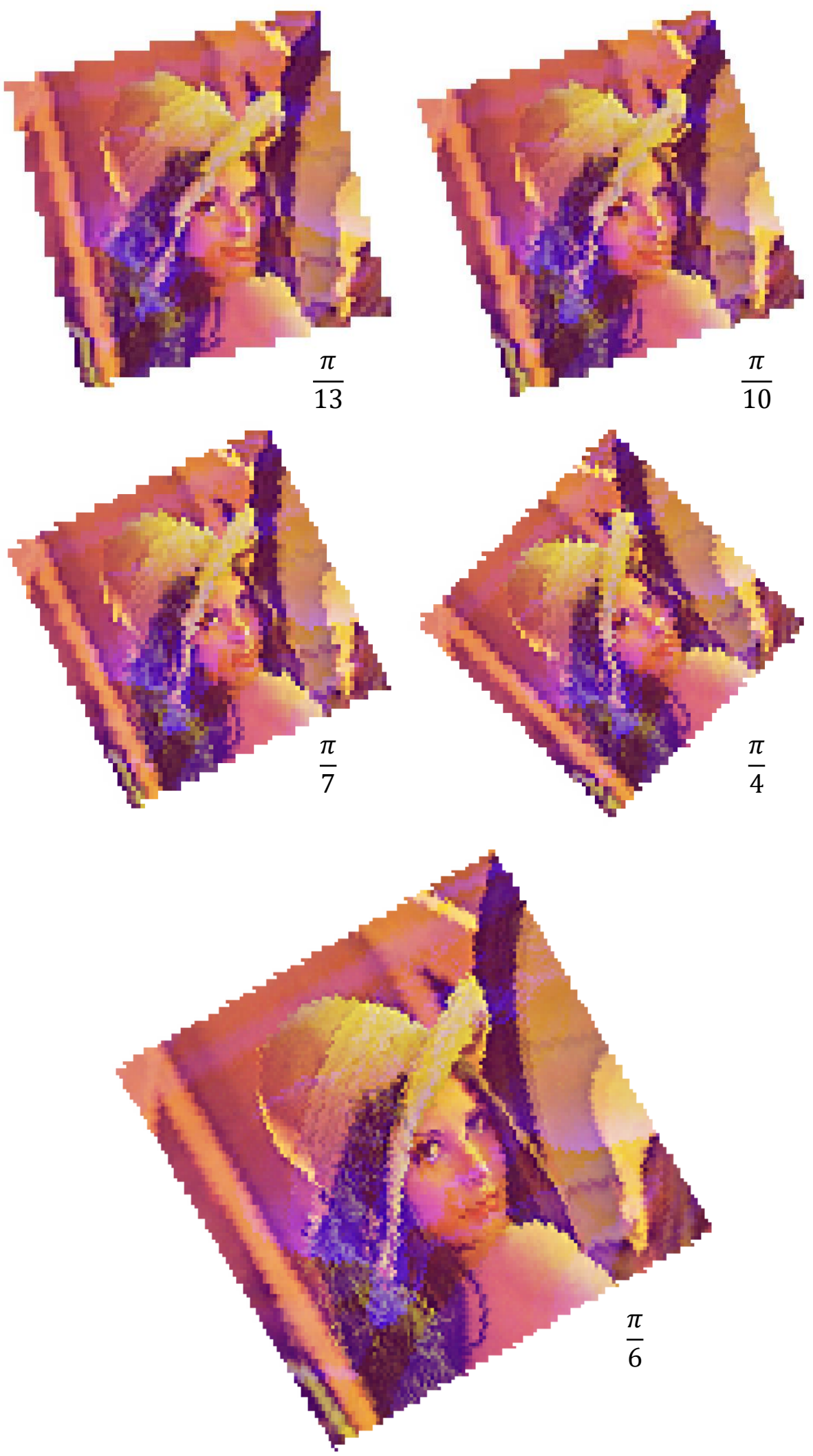

Fig. 6. Rotation of the Lena image by various angles and randomly chosen center. 